International Journal of Pure and Applied Mathematics

Volume 96 No. 1 2014, 135-141

ISSN: 1311-8080 (printed version); ISSN: 1314-3395 (on-line version)

url: http://www.ijpam.eu

doi: http://dx.doi.org/10.12732/ijpam.v96i1.10

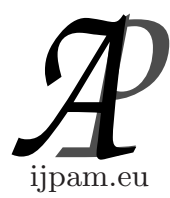

\title{
PI-1-1 NORMAL FORM IN A REGULAR CARDINAL
}

\author{
Martin Dowd \\ 1613 Wintergreen Pl. \\ Costa Mesa, CA 92626, USA
}

\begin{abstract}
A normal form is given for $\Pi_{1}^{1}$ formulas over $V_{\kappa}$ for a regular cardinal $\kappa$. Using it, a new characterization of weakly compact cardinals is given.
\end{abstract}

AMS Subject Classification: 03E55

Key Words: $\Pi_{1}^{1}$ normal form

\section{Formulas}

Normal form theorems for definable sets of reals are central to descriptive set theory. Here such will be considered for definable sets over $V_{\kappa}$ where $\kappa$ is a regular cardinal. In the case $\kappa=\omega$, the Ackerman coding [1] provides a definable bijection $V_{\omega} \mapsto \omega$, so $V_{\omega}$ could be used as the basic domain in this case.

Let $L_{\in}$ denote the first order language of set theory, with binary relations $=, \in$. Let $L_{\in}^{s}$ denote $L_{\in}$ with set variables added, considered as unary predicate symbols (so $X(x)$ is an atomic formula). Let $L_{\in}^{f}$ denote $L_{\in}$ with function variables added, considered as unary function symbols (so $F(x)$ is a term).

For each of these languages, a bounded quantifier is one of the form $\forall x \in t$ or $\exists x \in t$, where $t$ is a term (a variable in the case of $L_{\in}$ or $L_{\in}^{s}$ ), such that $x$ does not occur in $t$. A bounded, or $\Delta_{0}^{0}$, formula is a formula where all quantifiers 
are bounded. For $n \geq 1$ a $\Sigma_{n}^{0}\left(\Pi_{n}^{0}\right)$ formula is a $\Delta_{0}^{0}$ formula, preceded by $n$ alternating blocks of first-order quantifiers, the first of which is $\exists(\forall)$.

A $\Delta_{0}^{1}$ formula is a formula which is $\Delta_{0}^{0}, \Sigma_{n}^{0}$ for some $n$, or $\Pi_{n}^{0}$ for some $n$. For $n \geq 1$ a $\Sigma_{n}^{1}\left(\Pi_{n}^{1}\right)$ formula is a $\Delta_{0}^{1}$ formula, preceded by $n$ alternating blocks of second-order quantifiers, the first of which is $\exists(\forall)$. For $j=1,2$ the notation $\Sigma_{n}^{j s}$ is used for " $\Sigma_{1}^{j}$ over $L_{\in}^{s}$ ", etc.

Let $\operatorname{Pow}(x)$ denote the power set of $x$. Let $\mathcal{C}$ denote $\operatorname{Pow}\left(V_{\kappa}\right)$. Formulas of $L_{\in}^{s}$ may be considered as defining predicates in a Cartesian product of copies of $V_{\kappa}$ and $\mathcal{C}$ (a "product space"). Let $\mathcal{N}$ denote $\left(V_{\kappa}\right)^{V_{\kappa}}$. Formulas of $L_{\in}^{f}$ may be considered as defining predicates in a Cartesian product of copies of $V_{\kappa}$ and $\mathcal{N}$.

Say that a predicate is $\Sigma_{n}^{j}$ for $j=1,2\left(\Pi_{n}^{j}\right)$ if it is defined by a $\Sigma_{n}^{j}\left(\Pi_{n}^{j}\right)$ formula. By predicate logic:

- Any of these classes is closed under $\wedge$ and $\vee$.

- If $P$ is $\Sigma_{n}^{j}\left(\Pi_{n}^{j}\right)$ then $\neg P$ is $\Pi_{n}^{j}\left(\Sigma_{n}^{j}\right)$

- If $m>n$ then a $\Sigma_{n}^{j}$ or $\Pi_{n}^{j}$ predicate is both $\Sigma_{m}^{j}$ and $\Pi_{m}^{j}$.

Various other closure properties may be shown; see [3] for example.

The notion of a $\Delta_{n}^{j}$ predicate is defined in the usual manner, and by a standard abuse of language, a $\Delta_{n}^{j}$ formula is a $\Sigma_{n}^{j}$ or $\Pi_{n}^{j}$ formula which defines a $\Delta_{n}^{j}$ predicate.

\section{2. $L_{\in}^{\mathrm{S}}$ and $\boldsymbol{L}_{\in}^{\mathrm{f}}$}

Lemma 1. For any atomic formula $\phi$ of $L_{\in}^{f}$ there is a $\Delta_{1}^{0}$ formula $\phi^{\prime}$ of $L_{\in}^{s}$ such that $\phi\left(F_{1}, \ldots, F_{k}\right)$ iff $\phi^{\prime}\left(\gamma_{F_{1}}, \ldots, \gamma_{F_{k}}\right)$ where $\gamma_{F}$ is the graph of $F$.

Proof. In a standard manner, $t=u$ or $t \in u$ may be rewritten in a $\Delta_{1}^{0}$ form involving only atomic formulas of the form $y=F(x), w=x$, or $w \in x$. Now, $y=F(x)$ iff $\exists p\left(p=\langle x, y\rangle \wedge \gamma_{F}(p)\right)$ iff $\forall p\left(p=\langle x, y\rangle \Rightarrow \gamma_{F}(p)\right)$. Finally, $p=\langle x, y\rangle$ is $\Delta_{0}^{0}$.

Lemma 2. The predicate Func $(X)$ stating that $X$ is the graph of a function is $\Pi_{2}^{0 s}$.

Proof. The formula $\forall x \exists p \exists y(p=\langle x, y\rangle \wedge X(p))$ states that $X$ is total. The formula $\forall x \forall p_{1} \forall p_{2} \forall y_{1} \forall y_{2}\left(p_{1}=\left\langle x, y_{1}\right\rangle \wedge p_{2}=\left\langle x, y_{2}\right\rangle \wedge X\left(p_{1}\right) \wedge X\left(p_{2}\right) \Rightarrow p_{1}=p_{2}\right)$ states that $X$ is single-valued. 
Lemma 3. For any atomic formula $\phi$ of $L_{\in}^{s}$ there is a $\Delta_{0}^{0}$ formula $\phi^{\prime}$ of $L_{\in}^{f}$ such that $\phi\left(X_{1}, \ldots, X_{k}\right)$ iff $\phi^{\prime}\left(\chi_{X_{1}}, \ldots, \chi_{X_{k}}\right)$ where $\chi_{X}$ is the characteristic function of $X$.

Proof. $X(x)$ iff $\chi_{X}(x) \neq 0$.

Further facts can be stated; see for example theorems 15.XXV and 15.XXVI of $[6]$ in the case of $\omega$.

\section{Normal Form}

As in the case of $\omega$, a normal form is more useful for formulas of $L_{\in}^{f}$ (see [5] for example). In this case Skolem functions can be expressed in a simple manner. The notation $\vec{x}$ is used to denote a sequence $x_{1}, \ldots, x_{k}$ of variables; $\exists \vec{x}$ denotes $\exists x_{1} \ldots \exists x_{k}$, etc. A formula $\phi$ with free variables or parameters among $\vec{F}, \vec{x}$ may be denoted $\phi(\vec{F}, \vec{x})$.

Theorem 4. If $\phi$ is a $\Delta_{0}^{1 f}$ formula there is a $\Delta_{0}^{0 f}$ formula $\psi$ such that $\phi$ is equivalent to $\exists \vec{F} \forall \vec{x} \psi$ (in models of a suitable fragment of set theory for $L_{\in}^{f}$ ).

Proof. This is a refinement of the Skolem normal form theorem of Section 4.2 of [4]. Indeed, by this theorem $\phi$ can be written as $\exists F_{1} \cdots \exists F_{k} \forall x_{1} \cdots \forall x_{l} \psi_{1}$ where in $\psi_{1} F_{i}$ may have multiple arguments (where in fact $\psi_{1}$ can be taken as open).

This may be rewritten as $\exists \vec{F} \forall \vec{x} \forall \vec{y}\left(H_{1} \wedge \cdots \wedge H_{k} \Rightarrow \psi_{2}\right)$, where $H_{i}$ is $y_{i}=$ $F_{i}\left(\vec{x}_{i}\right), \vec{x}_{i}$ is a subsequence of $\vec{x}$, and $\psi_{2}$ is $\psi_{1}$ with $F_{i}\left(\vec{x}_{i}\right)$ replaced by $y_{i}$.

Let $H_{i}^{\prime}$ be $x_{j_{1}}=0 \wedge \cdots \wedge x_{j_{t}} \Rightarrow y_{i}=G_{i}(z)$, where the $x_{j}$ are those not occurring in $\vec{x}$. The formula may be transformed to $\exists \vec{G} \forall \vec{x} \vec{y} z\left(z=\left\langle x_{1}, \ldots, x_{l}\right\rangle \wedge\right.$ $\left.H_{1}^{\prime} \wedge \cdots \wedge H_{k}^{\prime} \Rightarrow \psi_{2}\right)$.

The models in which the theorem holds include $V_{\kappa}$ for $\kappa$ a cardinal.

Corollary 5. If $\phi$ is a $\Sigma_{1}^{1 f}$ formula there is a $\Delta_{0}^{0 f}$ formula $\psi$ such that $\phi$ is equivalent to $\exists \vec{F} \forall \vec{x} \psi$.

Proof. Transform the first order part to Skolem normal form, prepend the original existential function quantifiers, and proceed as in the proof of the theorem.

Corollary 6. If $\phi$ is a $\Pi_{1}^{1 f}$ formula there is a $\Delta_{0}^{0 f}$ formula $\psi$ such that $\phi$ is equivalent to $\forall \vec{F} \exists \vec{x} \psi$. 
Proof. Apply the preceding corollary to $\neg \phi$.

$\exists \vec{F} \forall \vec{x} \psi$ may be transformed to $\exists F \forall x \psi$. Proceeding as in the proof of theorem $4, \exists \vec{G} \forall \vec{x} \vec{y} z\left(z=\left\langle x_{1}, \ldots, x_{l}\right\rangle \wedge H_{1}^{\prime} \wedge \cdots \wedge H_{k}^{\prime} \Rightarrow \psi_{2}\right)$ may be further transformed to $\exists G \forall \vec{x} \vec{y} \vec{w} z v\left(v=G(z) \wedge v=\left\langle w_{1}, \ldots, w_{k}\right\rangle \wedge z=\left\langle x_{1}, \ldots, x_{l}\right\rangle \wedge H_{1}^{\prime \prime} \wedge\right.$ $\cdots \wedge H_{k}^{\prime \prime} \Rightarrow \psi_{2}$ ), where $H_{i}^{\prime \prime}$ is $x_{j_{1}}=0 \wedge \cdots \wedge x_{j_{t}} \Rightarrow y_{i}=w_{i}$. Finally, a formula $\forall \vec{x} \psi$ may be transformed to $\forall u \forall \vec{x}\left(u=\left\langle x_{1}, \ldots, x_{l}\right\rangle \Rightarrow \psi\right)$. The quantifier $\forall x_{i}$ may then be replaced by bounded quantifiers.

Suppose $\kappa$ is a regular cardinal, $\phi(\vec{F})$ is a $\Delta_{0}^{0 f}$ formula over $V_{\kappa}$ (possibly with first order parameters), and $u \in V_{\kappa}$. Let $\phi^{\dagger u}$ denote $\phi$ with each occurrence of $F_{i}$ replaced by $F_{i} \uparrow u$. This may be written in $\Delta_{1}^{0 f}$ form in a standard manner, as $\exists \vec{f}\left(f_{1}=F_{1} \uparrow u \wedge \cdots \wedge f_{k}=F_{k} \uparrow u \wedge \phi(\vec{f})\right)$ and $\forall \vec{f}\left(f_{1}=F_{1} \uparrow u \wedge \cdots \wedge f_{k}=\right.$ $\left.F_{k} \uparrow u \Rightarrow \phi(\vec{f})\right)$.

For a $\Delta_{0}^{0 f}$ formula $\psi$ with free variables $\vec{x}, \vec{F}$ a predicate $Q_{\psi}(u, \vec{x}, \vec{F})$ will be defined by recursion on $\psi$, which holds iff the arguments of function applications are all in $u$. For a term $t$ let $Q_{t}$ is $\vee_{s} s \in u$ where $s$ ranges over subterms other than $t . Q_{t=u}=Q_{t \in u}=Q_{t} \wedge Q_{u}, Q_{\neq \psi}=Q_{\psi}, Q_{\psi_{1} \wedge \psi_{2}}=Q_{\psi_{1} \vee \psi_{2}}=Q_{\psi_{1}} \wedge Q_{\psi_{2}}$, and $Q_{\exists x \in t \psi}=Q_{\forall x \in t \psi}=Q_{t} \wedge \forall x \in t Q_{\psi}$.

Lemma 7. Suppose $\kappa$ is a cardinal; then in $V_{\kappa}, Q_{\psi} \Rightarrow\left(\psi \Leftrightarrow \psi^{\lceil u}\right)$. If $\kappa$ is regular, $\exists u Q_{\psi}$.

Proof. The first claim follows by induction on $\psi$. The second claim does also, using regularity for the bounded quantifiers.

\section{Downsets}

The notion of a tree is central to descriptive set theory. The generalization to a cardinal $\kappa$ is better behaved if $\kappa$ is assumed to be regular. Let $\mathcal{A}=\left\{f \in V_{\kappa}: f\right.$ is a function $\}$; by the restriction on $\kappa$ if a function $f: x \mapsto V_{\kappa}$ where $x \in V_{\kappa}$ then $f \in V_{\kappa}$.

$\mathcal{A}$ is ordered by inclusion. A subset $D \subseteq \mathcal{A}$ is said to be a downset if, $f \in D$ and $g \subseteq f$ imply $g \in D$. If $D$ is a downset a branch of $D$ is a function $F \in \mathcal{N}$ such that $F \uparrow x \in D$ for all $x \in V_{\kappa}$. Let $[D]$ denote the set of branches of $D$. If $[D] \neq \emptyset$ say that $D$ is branched; otherwise $[D]$ is unbranched.

$\mathcal{A}$ may be generalized in a well-known manner. Let $\mathcal{A}^{(k)}=\left\{\vec{f} \in \mathcal{A}^{k}\right.$ : $\left.\operatorname{Dom}\left(f_{1}\right)=\cdots=\operatorname{Dom}\left(f_{k}\right)\right\}$ where $\operatorname{Dom}(f)$ denotes the domain of the function $f ; \operatorname{Dom}(\vec{f})$ may be written for the common domain. $\mathcal{A}^{(k)}$ may be ordered by "componentwise inclusion" $\vec{f} \subseteq_{c} \vec{g}$, which holds iff $f_{i} \subseteq g_{i}$ for all $i$. The 
definition of a downset is essentially unchanged, and a branch is a vector $\vec{F}$ which is a branch componentwise.

If $D$ is a downset in $\mathcal{A}^{k+l}$ and $\vec{G} \in \mathcal{N}^{l}$ let $D_{@ \vec{G}}=\left\{\vec{f}:\left\langle\vec{f}, G_{1}\left\lceil\operatorname{Dom}(\vec{f}), \ldots, G_{l} \uparrow\right.\right.\right.$ $\operatorname{Dom}(\vec{f})\rangle \in D\}$. Note that $D_{@ \vec{G}}$ is a downset.

A $\Delta_{0}^{0 f}$ formula $\psi(\vec{F})$ may be translated to a $\Delta_{0}^{1 f}$ formula $\psi^{\prime}(\vec{f})$ in a wellknown manner; by abuse of notation $\psi(\vec{f})$ will be written for this formula. The notation " $\vec{x} \in_{c} u$ " will be used for $x_{1} \in u \wedge \cdots \wedge x_{t} \in u$.

Theorem 8. Suppose $\kappa$ is a regular cardinal. Suppose $\phi$ is a formula $\forall \vec{F} \exists \vec{x} \psi(\vec{x}, \vec{F}, \vec{G})$ where $\psi$ is $\Delta_{0}^{0 f}$ (and for brevity has no first order parameters). Let

$$
D=\left\{\langle\vec{f}, \vec{g}\rangle \in \mathcal{A}^{k+l}: \forall \vec{x} \in_{c} \operatorname{Dom}(\vec{f})\left(Q_{\psi}(\operatorname{Dom}(\vec{f}), \vec{x}, \vec{f}, \vec{g}) \Rightarrow \neg \psi(\vec{x}, \vec{f}, \vec{g})\right)\right\} .
$$

Then $D$ is a downset, and in $V_{\kappa}$, $\phi$ holds iff $D_{@ \vec{G}}$ is unbranched.

Proof. It follows readily by induction on $\psi$ that if $\left\langle\vec{f}^{\prime}, \vec{g}^{\prime}\right\rangle \subseteq_{c}\langle\vec{f}, \vec{g}\rangle$ then $Q_{\psi}\left(\operatorname{Dom}\left(\overrightarrow{f^{\prime}}\right), \vec{x}, \vec{f}^{\prime}, \vec{g}^{\prime}\right) \Rightarrow Q_{\psi}(\operatorname{Dom}(\vec{f}), \vec{x}, \vec{f}, \vec{g})$. It then follows that $D$ is a downset. Using lemma 7 it follows readily that if $\phi$ is true then $D_{@ \vec{G}}$ is unbranched; and conversely.

\section{Weak Compactness}

It is a well-known fact that a cardinal $\kappa$ is weakly compact iff it is $\Pi_{1}^{1}$-indescribable, that is, for any $\Pi_{1}^{1 s}$ sentence $\phi$, if $\models_{V_{\kappa}} \phi$ then $\models_{V_{\lambda}} \phi$ for some regular cardinal $\lambda<\kappa$.

Theorem 9. For a regular cardinal $\kappa$ the following are equivalent.

a. For any $\Pi_{1}^{1 f}$ sentence $\forall F \psi(F, G)$, if $\models_{V_{\kappa}} \forall F \psi(F, G)$ then for some regular cardinal $\lambda<\kappa, G \cap V_{\lambda}$ is total and $\models_{V_{\lambda}} \forall F \psi\left(F, G \cap V_{\lambda}\right)$.

b. For any $\Pi_{1}^{1 f}$ sentence $\forall F \psi(F, G)$ where $G \cap V_{\lambda}$ is total for all regular cardinals $\lambda<\kappa$, if $\models_{V_{\kappa}} \forall F \psi(F, G)$ then for some regular cardinal $\lambda<\kappa$, $\models_{V_{\lambda}} \forall F \psi\left(F, G \cap V_{\lambda}\right)$.

c. $\kappa$ is weakly compact.

Proof. $\mathrm{a} \Rightarrow \mathrm{b}$ is immediate. Suppose b holds. Suppose $\phi$ is a $\Pi_{1}^{1 s}$ formula $\forall X \psi(X, Y)$. Let $\phi^{\prime}$ be $\forall F \psi^{\prime}\left(F, \chi_{Y}\right)$ where $\psi^{\prime}$ is the translation of $\psi$ obtained using lemma 3. That $\mathrm{b} \Rightarrow \mathrm{c}$ follows, noting that $\chi_{Y} \cap V_{\lambda}$ is total.

Suppose $\kappa$ is weakly compact. Given $\phi=\forall F \psi(F, G)$, let $\phi^{\prime}$ be $\operatorname{Tot}\left(\gamma_{G}\right) \wedge$ $\forall X\left(\operatorname{Func}(X) \Rightarrow \psi^{\prime}\right)$ where $\psi^{\prime}$ is the translation of $\psi$ obtained using lemma 1 , 
Func is as in lemma 2, and $\operatorname{Tot}(X)$ is the first order predicate " $X$ is total". Then $\models_{V_{\kappa}} \phi^{\prime}$, so $\models_{V_{\lambda}} \phi^{\prime}$ for some $\lambda$, so $G \cap V_{\lambda}$ is total and $\models_{V_{\lambda}} \phi$. Thus, $\mathrm{c} \Rightarrow \mathrm{a}$.

Say that a regular cardinal $\kappa$ has the downset property iff, whenever $D \subseteq \mathcal{N}$ is an unbranched downset, there is a regular cardinal $\lambda<\kappa$ such that $D \cap V_{\lambda}$ is unbranched.

Theorem 10. If a regular cardinal $\kappa$ has the downset property then $\kappa$ is weakly compact.

Proof. Suppose $\phi$ is a $\Pi_{1}^{1 f}$ formula, which may be assumed to be in normal form $\forall \vec{F} \exists \vec{x} \psi$ where $\psi$ is $\Delta_{1}^{1 f}$, has no first order parameters, and for a second order parameter $G$ and a regular cardinal $\kappa G \cap V_{\lambda}$ is total. Suppose $\phi$ is true in $V_{\kappa}$. Then the downset $D_{@ \vec{G}}$ of theorem 8 is unbranched. By hypothesis $D_{@ \vec{G}} \cap V_{\lambda}$ is unbranched for some regular cardinal $\lambda<\kappa$. By theorem $8 \phi$ is true in $V_{\lambda}$.

Theorem 11. If $\kappa$ is a weakly compact cardinal then $\kappa$ has the downset property.

Proof. There is a $\Pi_{1}^{1 f}$ sentence in the parameter $D$ which is true in $V_{\lambda}$ for a regular cardinal $\lambda$ iff $D$ is an unbranched downset.

\section{Concluding Remarks}

It is a question of considerable interest, whether the existence of weakly compact cardinals can be justified by postulating sufficiently long stationary set chains (see[2]). Although the results given here do not shed much light on the problem, they do indicate that methods from descriptive set theory can be adopted to some extent. This suggests that further research in this area might be of interest.

\section{References}

[1] http://en.wikipedia.org/wiki/Ackermann_coding

[2] M. Dowd, Function Chains from Uniform Sigma-1-1 Well Orders, Int. J. Pure Appl. Math. , 93 no. 5 (2014), 661-684, http://dx.doi.org/10.12732/ijpam.v93i5.7 
[3] F. Drake, Set Theory, An Introduction to Large Cardinals, North-Holland, Netherlands (1974).

[4] H. Enderton, A Mathematical Introduction to Logic, Academic Press, USA (1972).

[5] A. Kanamori, The Higher Infinite, Springer-Verlag, Germany (2003).

[6] H. Rogers, Theory of Recursive Functions and Effective Computability, McGraw-Hill, USA (1967). 
